EPJ Web of Conferences 116, 01002 (2016)

DOI: $10.1051 /$ epjconf/201611601002

(C) Owned by the authors, published by EDP Sciences, 2016

\title{
The KM3NeT Digital Optical Module
}

\author{
Daniele Vivolo $^{1,2}$ for the KM3NeT Collaboration \\ ${ }^{1}$ University of Naples "Federico II", Department of Physics, via Cintia, 80126 Naples, Italy \\ ${ }^{2}$ INFN - Istituto Nazionale di Fisica Nucleare, Section of Naples, via Cintia, 80126 Naples, Italy
}

\begin{abstract}
KM3NeT is a European deep-sea multidisciplinary research infrastructure in the Mediterranean Sea. It will host a km3-scale neutrino telescope and dedicated instruments for long-term and continuous measurements for Earth and Sea sciences. The KM3NeT neutrino telescope is a 3-dimensional array of Digital Optical Modules, suspended in the sea by means of vertical string structures, called Detection Units, supported by two prestretched Dyneema ropes, anchored to the seabed and kept taut with a system of buoys. The Digital Optical Module represents the active part of the neutrino telescope. It is composed by a 17 -inch, $14 \mathrm{~mm}$ thick borosilicate glass (Vitrovex) spheric vessel housing 31 photomultiplier tubes with 3-inch photocathode diameter and the associated front-end and readout electronics. The technical solution adopted for the KM3NeT optical modules is characterized by an innovative design, considering that existing neutrino telescopes, Baikal, IceCube and ANTARES, all use large photomultipliers, typically with a diameter of 8 " or $10 "$. It offers several advantages: higher sensitive surface $\left(1260 \mathrm{~cm}^{2}\right)$, weaker sensitivity to Earth's magnetic field, better distinction between single-photon and multi-photon events (photon counting) and directional information with an almost isotropic field of view. In this contribution the design and the performance of the KM3NeT Digital Optical Modules are discussed, with a particular focus on enabling technologies and integration procedure.
\end{abstract}

\section{Introduction}

The KM3NeT project aims at the realization of a deep-sea multidisciplinary research infrastructure in the Mediterranean Sea. In particular, a multi- $\mathrm{km}^{3}$-scale neutrino telescope, with dedicated instruments for long-term and continuous measurements in earth and sea sciences, is currently under construction. The KM3NeT research facility will be realized as an installation distributed over several sites, with common detector technology, management, data handling and operation control. In particular, a large volume neutrino telescope, called ARCA (Astroparticle Research with Cosmics in the Abyss), will be installed in the Italian site of Portopalo di Capopassero in Sicily, while a denser and more compact version of the detector, ORCA (Oscillation Research with Cosmics in the Abyss) in the KM3NeT-Fr site of Toulon (France), will be dedicated to the study of low energy atmospheric neutrino oscillations [1].

$\mathrm{KM} 3 \mathrm{NeT}$ is a three-dimensional array of optical detectors contained in glass spheres that are designed to resist the hostile conditions (high hydrostatic pressure and water salinity) of the deepsea environment. Such modules, called Digital Optical Modules (DOMs), are suspended in the sea by means of vertical string structures, called Detection Units (DUs), supported by two pre-stretched

This is an Open Access article distributed under the terms of the Creative Commons Attribution License 4.0, which permits unrestricted use, distribution, and reproduction in any medium, provided the original work is properly cited. 
Dyneema ropes, anchored to the seabed and kept taut by a system of buoys. Each DU is equipped with 18 DOMs, for a total length of more than $700 \mathrm{~m}$, and hosts the optical detectors readout electronics and all the devices required for calibration, positioning and acoustics measurements as well as sea and Earth science instrumentation like video cameras, conductivity-temperature-depth probes, Doppler current profilers, chemical analysers and seismographs [2].

Currently, the KM3NeT collaboration has started the construction of the first phase of the project, consisting of 24 DUs of the ARCA detector and 7 DU of ORCA.

\section{The Digital Optical Module}

The Digital Optical Module represents the active part and hence the real heart of KM3NeT.

An exploded view is shown in Fig. 1. The DOM is composed by a 17-inch, $14 \mathrm{~mm}$ thick borosilicate glass (Vitrovex) spheric vessel housing 31 PMTs with 3-inch photocathode diameter, the associated frontend and readout electronics, calibration components and accessory instrumentation for monitoring purposes. The vessel provides an adequate mechanical resistance to the extreme compressive stresses of deep-sea environment (the ambient hydrostatic pressure is in the range of 250-400 bar) while keeping a transparency of more than $95 \%$ at a wavelength of $\lambda=350 \mathrm{~nm}$.

The multi-PMT solution, which had already been considered in the framework of the DUMAND project [3], represents one of the main differences of the KM3NeT DOM with respect to the standard concept of optical modules in large-volume neutrino telescopes, which are traditionally equipped with single large PMTs.

First of all, an overall sensitive surface of $1260 \mathrm{~cm}^{2}$ is achieved, thus optimizing the detection efficiency. This is a crucial factor, considering the extremely low interaction rate of astrophysical neutrinos. Small surface PMTs exhibit a weak sensitivity to Earth's magnetic fields, with the appealing consequence that no shielding is required and hence integration complexity and costs are reduced. A segmented detection surface makes possible an improved distinction between single-photon from multi-photon events. Indeed, in a DOM a two-photon event can be unambiguously recognized if the two photons hit separate tubes, a condition that occurs in $85 \%$ of cases for photons arriving from the same direction. Moreover, the almost isotropic angular coverage of the PMTs, which are oriented from vertically downwards to 58 deg upwards, permits the measure of unscattered light from sources with arbitrary orientation from the DOMs [4].

In addition, a strong reduction of the failure risk is achievable. Indeed, for a small diameter device the integrated anode charge is significantly smaller, thus making it less subject to ageing and decreasing its failure rate to something around $10^{-4}$ per year.

\subsection{Mechanics}

A nylon support structure, manufactured by 3D printing with the selective laser sintering method, has the function to hold the 31 PMTs inside the DOM, defining their positions, their spatial orientations and their distances from the glass vessel. All the PMT locations are tapered and a silicone O-ring tightly seals around the PMT just below its head [4].

The DOM vessel is made of two half spheres. The lower hemisphere houses 19 PMTs, power board and readout electronics, a LED flasher pointing upwards for timing calibration between DOMs (called nanobeacon, see Fig. 1) and a pressure gauge to monitor the air pressure inside the DOM before deployment. The upper one contains 12 PMTs and an acoustic sensor attached to the glass sphere for positioning purposes. The photomultipliers are glued to the DOM glass vessel by means of a twocomponents transparent silicon optical gel (Wacker 612), filling the cavity between the support structure and the glass, and assuring optical contact. In order to reduce the unwanted reflections, a refractive index of $\approx 1.40$ is required, close to both the refractive indexes of the glass vessel (1.47) and of the PMT 


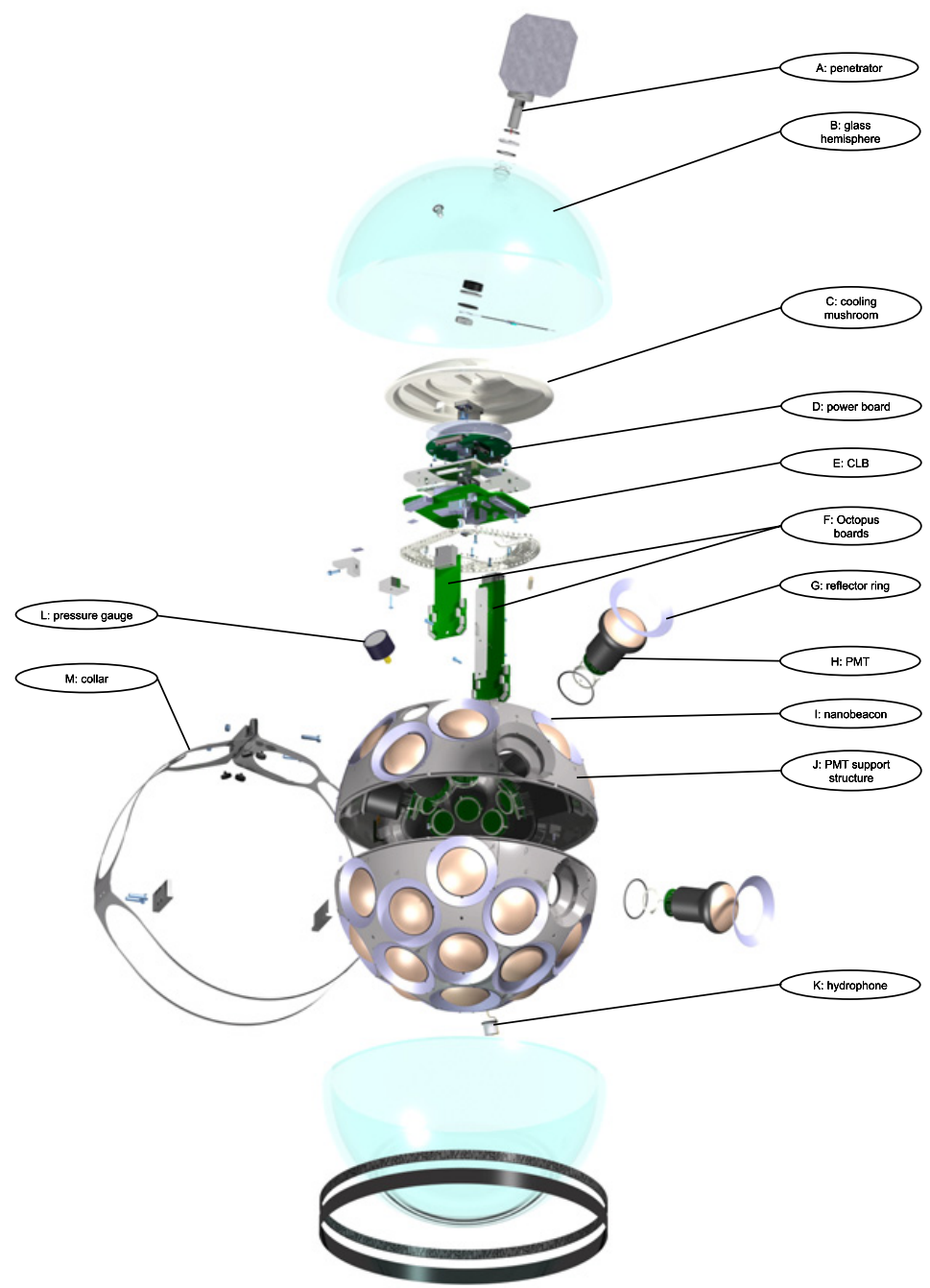

Figure 1. Exploded view of a KM3NeT DOM. The main components are indicated and referenced in the text.

window (1.51-1.54), with an attenuation length greater than $40 \mathrm{~cm}$ for wavelengths above $350 \mathrm{~nm}$. In addition, sufficiently elastic support structure and gel provide a precious help in the absorption of the shocks and the vibrations induced by transportation and deployment, contributing to accommodate the shrinkage of the glass vessel under the high hydrostatic pressure.

The DOM contains also a passive cooling system, based on the heat conduction mechanism, aimed at keeping the temperature of the electronic components as low as possible, thus maximizing their lifetime. In order to optimize the transfer of the heat generated by the electronics to the seawater, a metallic structure is required, with a large contact surface with the inner surface of the glass vessel. The cooling system is made by a mushroom-shaped Aluminium structure coupled to an Aluminium bar and is dimensioned to maintain temperatures below $30^{\circ} \mathrm{C}$ for an overall power dissipation inside the glass vessel of up to $20 \mathrm{~W}$. 


\subsection{Photomultiplier tubes}

The choice of the optical sensors is crucial. The overall performance of the telescope, in terms of efficiency and track reconstruction resolution, is intrinsically related to the basic performance of the adopted photodetectors. The adopted solution is represented by a 3-inch PMT. In particular, the PMTs used in the current KM3NeT phase are the Hamamatsu R12199-02.

In order to meet the KM3NeT requirements, the PMTs need to fulfil several requisites [2], including some geometry constraints. The reduced space available inside the DOM imposes an overall limitation on the length of the device (less than $122 \mathrm{~mm}$ ), while the outer convex surface of the PMT must match as precisely as possible the curvature of the inner surface of the glass sphere.

The performance specifications for the PMTs are summarized in the following. High quantum efficiency is required (>23\% at $404 \mathrm{~nm}$ and $>20 \%$ at $470 \mathrm{~nm}$ ), with an inhomogeneity of cathode response of less than $10 \%$. Taking into account the noise level of the electronic circuit for PMT readout, the value of the gain of each photomultiplier is fixed at $3 \times 10^{6}$, with a peak-to-valley ratio of at least 3 for an acceptable resolution. Considering that for a 3-inch PMT the optical background in the sea water is of the order of few tens of $\mathrm{kHz}$, the dark count rate of the adopted PMTs (given by the combination of thermal electron emission from photocathode and dynode chain and radioactive decay in the glass and in the optical gel, mainly in the form of ${ }^{40} \mathrm{~K}$ decay) must be less than $3 \mathrm{kHz}$.

Since the time resolution of the optical sensor has a crucial impact on the reconstructed muon track angular resolution, a small Transit Time Spread is preferable, compared with the timescale of the absolute time calibration of the telescope. For the KM3NeT case, a TTS of $2 \mathrm{~ns}$ (expressed in terms of the standard deviation $\sigma$ ) has been set as an upper limit.

\section{DOM integration}

DOM integration is a delicate process, managed by a dedicated procedure. All the design features are optimized for functionality and all the components must pass characterization tests and fulfil acceptance criteria before use.

The two hemispheres of the DOM are constructed separately. At the end of the integration process, the DOM is closed connecting the two halves and making a slight underpressure in the sphere $(0.2$ bar below atmospheric pressure). Also the support structure is segmented in two halves, each one made of two parts. After the 3-D printing process the structures are white, therefore, in order to reduce the optical background they must be painted black before being glued together.

After mounting the PMTs in the support structure, they are connected to frontend and readout electronics and then glued to the glass vessel. In order to transmit the optical data through the fibre leading to the outside of the DOM, a splicing of optical fibres is required.

During the integration process, several tests are performed: the mounted penetrator is subject to a helium leak test, the fibre splice and resulting optical power are measured and a functional test of all systems is performed before applying the optical gel [2]. After the DOM closure, an extensive final acceptance test is performed in a light-tight dark box.

\section{Conclusions}

The multi-PMT solution represents one of the main differences of the KM3NeT DOM with respect to the standard concept of optical modules in large-volume neutrino telescopes, which are traditionally equipped with single large PMTs, providing strong advantages in terms of photon counting, background rejection capabilities and direction sensitivity.

After a dedicated prototyping program, aimed at validating its design and performance, the KM3NeT collaboration has started a mass production of DOMs for both ARCA and ORCA detectors in four DOM integration sites. 
Very Large Volume Neutrino Telescope (VLVnT-2015)

\section{References}

[1] R. Coniglione, these proceedings (2015)

[2] R. Bruijn and D. van Eijk (for the KM3NeT collaboration), PoS(ICRC2015), 1157

[3] V.V. Borog et al., Proceedings of the 16th ICRC, 10, 380-384

[4] KM3NeT Technical Design Report, http://www.km3net.org/TDR/TDRKM3NeT.pdf 